\title{
Rolling based Energy Storage System Operation Strategies Considering Wind Power Forecast Uncertainty
}

\author{
Eunsung Oh \\ Department of Electrical and Electronic Engineering, Hanseo Unversity, Chungcheongnam-do 31962 South \\ Korea.esoh@hanseo.ac.kr
}

\begin{abstract}
This paper propose a rolling base energy storage system (ESS) operation strategy considering wind power forecasting uncertainty. ESS is worked according to operational time horizon $(\mathrm{OTH})$. However, the wind power uncertainty characteristic is changed to ahead time of forecasting. For an effective ESS operation, ESS rolling is required. This paper firstly formulates the optimization problem for the ESS operation to manage the uncertainty, and presents the operation and rolling strategies applying Lagrangian relaxation considering OTH and rolling time horizon (RTH). Numerical results show that the proposed method effectively manages the uncertainty, and suggest that the OTH and RTH should be balanced to optimal ESS operation.
\end{abstract}

Key words : Energy storage, forecast, intra-day, rolling, uncertainty, wind power.

\section{INTRODUCTION}

Renewable based energy generation is steadily increasing due to its clean and sustainable nature. In 2017 , renewable power grew by $17 \%$ contributed about $50 \%$ of the global power generation growth [1]. It is estimated that renewables capture more than $65 \%$ of global investment in power plants to 2040 [2]. In particular, wind power generation (WPG) leads the expansion of renewables. WPG provided more than 50\% of renewables growth [1] and supplied about $12 \%$ of power in the European Union [3].

WPG implies risks due to its intermittent and stochastic characteristics. To support the system, WPG forecasting is required for reducing the risk and increasing the utility of the use. Various forecasting methods have been performed [4-7]. The authors in [4] reviewed very short-term WPG forecasting techniques classified into numerical weather prediction (NWP)-physical or statistical-artificial intelligence (AI) methods. It is shown that statistical-AI methods has lower prediction errors in very short-term forecasting for horizons until $6 \mathrm{~h}$ ahead. An multi-model methodology blending multiple single-algorithm is proposed with a deep feature selection process [5]. The algorithm performs about $4 \%$ normalized mean absolute error (NMAE) in $1 \mathrm{~h}$ ahead

This work was supported by 2018 Research Grant of Hanseo University.

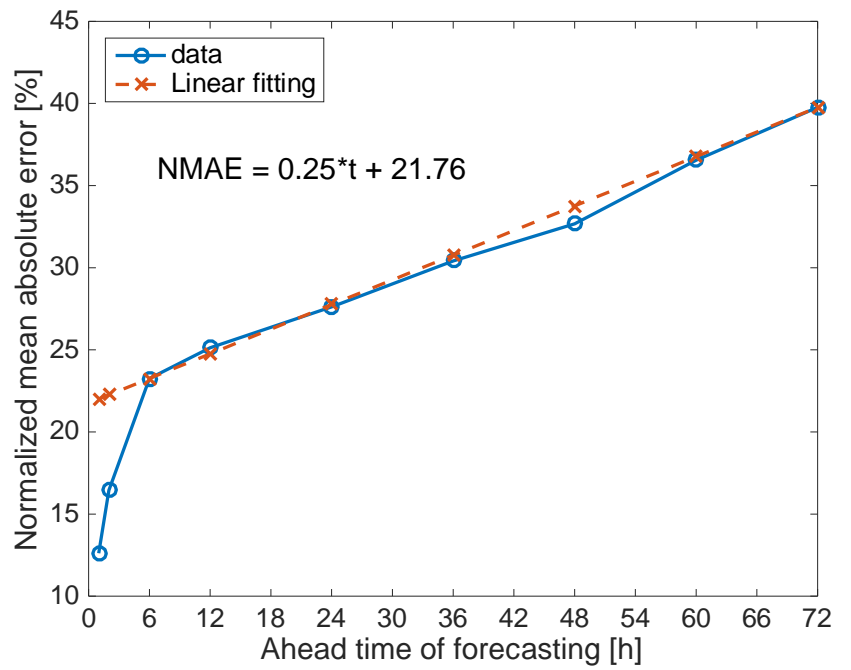

Figure 1: Relationship between the ahead time and NMAE of the WPG forecasting.

forecasting horizon. In [6], a NWP based forecasting method is presented using additional domain knowledge and historical data training. It shows about 6\% NMAE in 24 hour ahead forecasting horizon. A scalable graph deep learning based wind speed forecasting is proposed considering the localized first-order approximation of spectral graph convolutions and the temporal long short-term memory (LSTM) network features of the graph nodes in [7]. It averagely presents from $10 \%$ NMAE in $1 \mathrm{~h}$ ahead forecasting horizon to $20 \%$ NMAE in $24 \mathrm{~h}$ ahead forecasting horizon. The accuracy of forecasting techniques has improved. However, it is impossible to completely resolve the uncertainty of WPG because of the chaotic and randomness of wind.

Energy storage system (ESS) is a key technology to manage the WPG uncertainty [8]. ESS is operated as a buffer during the energy imbalance between actual generation and forecasting. Larger ESS capacity manages more uncertainty, but ESS cost is expensive [9]. Therefore, ESS techniques can be classified into sizing and operation. The influence of WPG forecast error is described on the EES sizing with a wind farm in France [10]. A supercapacitor-batteries hybrid-ESS sizing is developed using the statistical information for WPG smoothing in [11]. The authors in [12] presents iterative search based ESS sizing algorithms considering micro-grid reliability and system cost. Iterative ESS sizing algorithm is also proposed with diesel generator and its fuel cost [13]. In 


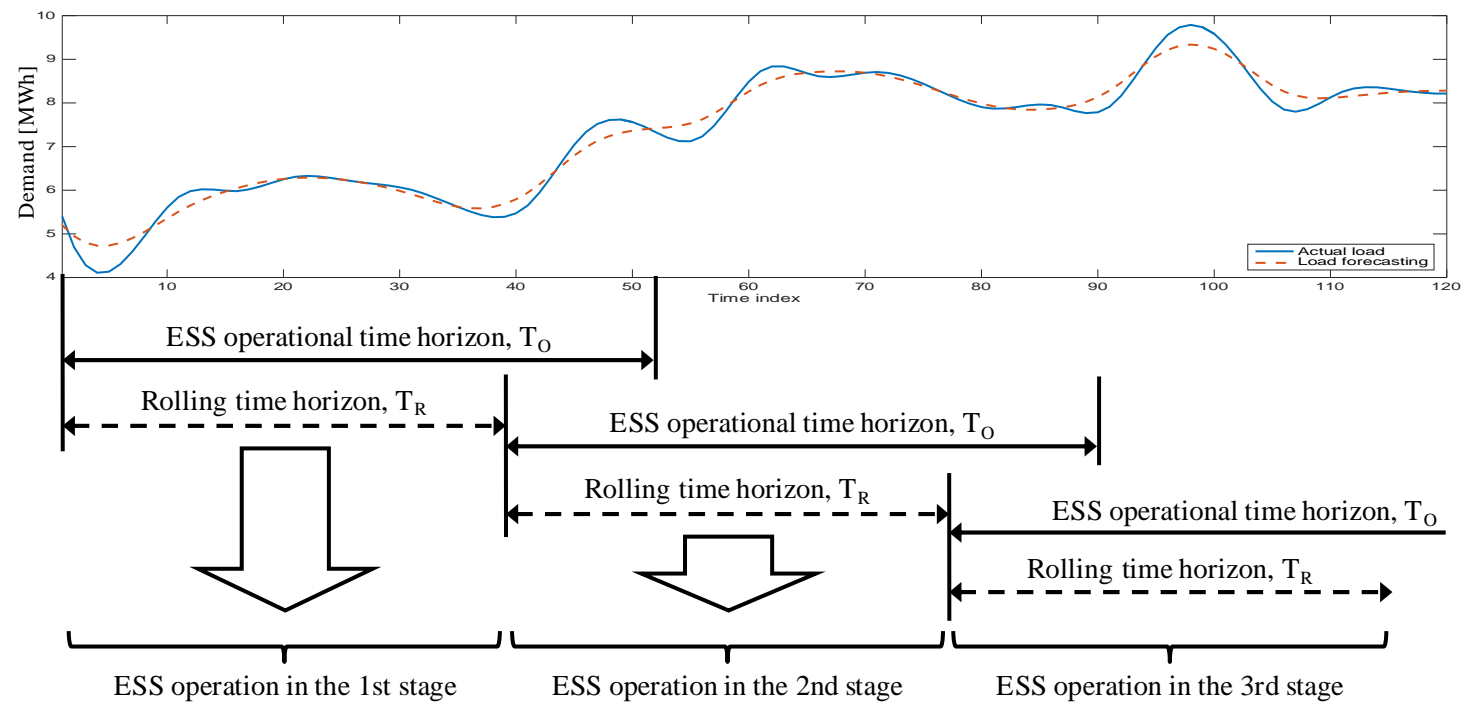

Figure 2: Rolling-based ESS operation framework.

[14], discrete Fourier transform based ESS sizing is proposed cutting-off high-frequency component to control the WPG uncertainty. These studies determine the EES size in the form of managing the forecast error within a certain range. Detailed error management is achieved through ESS operation.

ESS operation is focused on the enhancement of ESS effectiveness within the ESS size. The authors in $[15,16]$ present dynamic programming based ESS operations to shift WPG for maximizing the daily profit. In [17], a coordinated operational dispatch scheme for a wind farm with a battery ESS is proposed to improve the wind farm dispatchability by reducing the forecast imbalance. Meta heuristic approach based algorithms such as particle swarm optimization (PSO) [18], genetic algorithm (GA) [19], and hybrid algorithms mixed two or three approach $[20,21]$ are suggested for managing the WPG uncertainty. Most of EES operation is considered the ESS size as a main constraint. Because the EES size is limited the operational availability. However, with the accumulated and propagated characteristics of the WPG uncertainty, the operational time horizon restricts the ESS operational availability. To reduce this restriction, rolling based ESS operation method has been proposed considering reliability [22] and system profit [23]. In energy management system, the intra-day operation is applied as a rolling scheme for effective unit commitment considering microgrid [24] and renewables [25]. However, it is required considering both forecasting and ESS operation [26, 27].

This study focuses on an effective rolling based ESS operation strategy for managing the wind power forecasting uncertainty. Firstly the ESS operation problem is formulated considering the ESS operational time horizon (OTH) as an optimization problem. To solve the problem, the Lagrangian relation is applied. Using that, the ESS operation strategy is proposed related to the ESS rolling time horizon (RTH). The strategy is decided according to Lagrangian multipliers that presented the characteristics of the uncertainty and ESS. Numerical results with the actual wind power generation and its forecasting show that the proposed method can enhance the ESS operation performance by reducing the uncertainty, and it is required the balance between OTH and RTH for the effective ESS operation.

\section{METHODOLOGY}

\subsection{Rolling-based ESS Operation Framework}

The framework provides the ESS operational scheduling according to RTH and OTH. The ESS operation is optimized to take into account OTH longer than RTH. The solution in RTH is applied as the ESS operational scheduling and its residual outcome is combined with new WPG forecasting to produce the input to the next stage re-scheduling. The rolling based ESS operation framework is presented in Figure 2.

An ESS operation problem is solved every OTH considering the WPG forecasting. The output of the problem is the ESS operational scheduling such as ESS charge or discharge quantity at each time. The subset of the solution in RTH is then completed by deciding the operational scheduling and applied. At each time, the ESS is operated considering the scheduling and the actual WPG.

\subsection{Operation Method}

In this work, the goal of the ESS operation is to enhance the WPG system reliability by managing the uncertainty. The uncertainty is arisen by the WPG forecast error. Therefore, the objective of the ESS operation problem is modeled as an error function according to the WPG forecasting such as NMAE [5 - 7].

Let $q_{t}$ be the ESS operation during OTH, $\mathcal{T}_{O}=$ $\left\{1, \cdots, t, \cdots, T_{O}\right\}$. The objective function is written as

$$
\theta\left(\mathrm{q}_{\mathrm{t}}\right)=\frac{1}{T_{O}} \sum_{\mathrm{t} \in \mathcal{T}_{O}}\left|\frac{g_{t}-\hat{g}_{t}-q_{t}}{\mathrm{~g}_{\mathrm{t}}}\right|,
$$

where $g_{t}$ and $\hat{g}_{t}$ be the actual WPG and the WPG 
forecasting at time $t$. However, the information about the actual WPG $g_{t}$ cannot be achieved in causal systems [28]. Therefore, the function in (1) is changed to

$$
\theta\left(\mathrm{q}_{\mathrm{t}}\right)=\frac{1}{T_{O}} \sum_{\mathrm{t} \in \mathcal{T}_{O}} \frac{\left|e_{t}-q_{t}\right|}{\hat{g}_{t}},
$$

where $e_{t}=\mathbb{E}\left\{g_{t}-\hat{g}_{t}\right\}$ is the expected WPG forecast error.

The function in (2) is a special case of $l_{1}$ norm. To apply the mathematical operation such as differentiation, it is relaxed as [29]

$$
\tilde{\theta}\left(\mathrm{q}_{\mathrm{t}}\right)=\frac{1}{T_{O}} \sum_{\mathrm{t} \in \mathcal{T}_{O}} \frac{\left(e_{t}-q_{t}\right)^{2}}{2 \widehat{g}_{t}},
$$

The ESS size constraints the ESS operation, as follow;

$$
\begin{aligned}
& -E_{P S} \leq q_{t} \leq E_{P S}, \quad t \in \mathcal{T}_{O}, \\
& E_{E S}^{M I N} \leq s_{t} \leq E_{E S}^{M A X}, t \in \mathcal{T}_{O},
\end{aligned}
$$

where $s_{t}$ is the ESS state-of-charge (SoC) calculated as $s_{t}=q_{t}+s_{t-1}$, and $s_{0}$ becomes the initial state of ESS. The first constraint in (4a) expresses the ESS power subsystem (PS) constraint related to power conditional system (PCS). It limits the maximum charge/discharge power at time $t$ as $E_{P S}$. The second constraint in (4b) is the ESS energy subsystem (ES) constraint. The bound of the minimum/maximum SoC, $E_{E S}^{M I N}$ and $E_{E S}^{M A X}$ is decided considering the ESS characteristics such as material of ES and life time.

Using that, the ESS operation problem is formulated as

$$
\begin{array}{cc}
\min _{\mathrm{q}_{\mathrm{t}}} & \tilde{\theta}\left(\mathrm{q}_{\mathrm{t}}\right) \\
\text { s.t. } & (4 a),(4 b) .
\end{array}
$$

The problem in (5) is a quadratic problem. Therefore, the Lagrangian relaxation is applied to solve the problem [30],

$$
\begin{array}{r}
f=\tilde{\theta}\left(\mathrm{q}_{\mathrm{t}}\right)-\sum_{t \in \mathcal{T}_{O}} \lambda_{t}^{-}\left(q_{t}+E_{P S}\right)+\sum_{t \in \mathcal{T}_{O}} \lambda_{t}^{+}\left(q_{t}-E_{P S}\right) \\
-\sum_{t \in \mathcal{T}_{O}} v_{t}^{-}\left(s_{t}-E_{E S}^{M I N}\right)+\sum_{t \in \mathcal{T}_{O}} v_{t}^{+}\left(s_{t}-E_{E S}^{M A X}\right),
\end{array}
$$

where $\lambda_{t}^{-}, \lambda_{t}^{+}, v_{t}^{-}$, and $v_{t}^{+}$are Lagrangian multipliers that have zero or positive values [29]. The Lagrangian multipliers superscripted with minus (-) and plus (+) express the effect of ESS discharge and charge operation, respectively.

The Lagrangian multipliers and the optimal solution should satisfy the Karush-Kuhn-Tucker (KKT) condition,

$$
\begin{aligned}
& \frac{\partial f}{\partial q_{t}}=\frac{\partial \widetilde{\theta}\left(q_{t}\right)}{\partial q_{t}}+\left(\lambda_{t}^{+}-\lambda_{t}^{-}\right)+\sum_{i=t}^{T_{0}}\left(v_{i}^{+}-v_{i}^{-}\right)=0, \\
& \lambda_{t}^{-}\left(q_{t}+E_{P S}\right)=0, \lambda_{t}^{+}\left(q_{t}-E_{P S}\right)=0, t \in \mathcal{T}_{O}, \\
& v_{t}^{-}\left(s_{t}-E_{E S}^{M I N}\right)=0, v_{t}^{+}\left(s_{t}-E_{E S}^{M A X}\right)=0, t \in \mathcal{J}_{O} .
\end{aligned}
$$

From (7a), the optimal ESS operation $q_{t}^{*}$ is calculated as

$$
q_{t}^{*}=\underbrace{e_{t}}_{\text {Error part }}-\underbrace{T_{o} \hat{g}_{t}\left(\lambda_{t}^{+}-\lambda_{t}^{-}\right)}_{\text {PS part }}-\underbrace{T_{o} \hat{g}_{t} \sum_{i=t}^{T o}\left(v_{i}^{+}-v_{i}^{-}\right)}_{\text {ES part }} .
$$

and, it is constructed as three parts,

- Error part: This is the part to compensate the error between actual WPG and WPG forecasting.

- PS part: This part modifies the ESS operation considering the PS constraint in (4a). From the condition in (7b), the Lagrangian multipliers $\lambda_{t}^{-}$and $\lambda_{t}^{+}$has a value only when the ESS operation touches the PS constraint. As shown the results in [14], the sufficient size of PS is smaller than the ES size, and a larger size of PCS is installed in preparation for expansion [31]. Therefore, this part by $\lambda_{t}^{-}$and $\lambda_{t}^{+}$ could be neglected as zero.

- ES part (Residual part): Similar to the PS part, this part also adjusts the ESS operation considering the ES constraint in (4b). The feature of this part is that it considers not only the current operation but also the future operation. This part limits the current operation to prepare the onward operation, so it is said the residual part. The Lagrangian multipliers $v_{t}^{-}$ and $v_{t}^{+}$are recursively measured from (7c) and (8).

\subsection{Rolling Method}

In the rolling based ESS operation, the solution in RTH is used for the actual ESS operation. Therefore, NMAE is modified from (2) related to the rolling time $T_{R}$,

$$
\Theta\left(T_{R}\right)=\frac{1}{T_{R}} \sum_{\mathrm{t} \in \mathcal{T}_{R}} \frac{\left|e_{t}-q_{t}^{*}\right|}{\hat{g}_{t}},
$$

where $\mathcal{T}_{R}=\left\{1, \cdots, t, \cdots, T_{R}\right\}$.

Applying the optimal solution in (8), it is calculated as

$$
\Theta\left(T_{R}\right)=\frac{T_{\mathrm{O}}}{T_{R}} \sum_{\mathrm{t} \in \mathcal{T}_{R}}\left|\left(\lambda_{t}^{+}-\lambda_{t}^{-}\right)+\sum_{i=t}^{T_{O}}\left(v_{i}^{+}-v_{i}^{-}\right)\right| .
$$

Assuming that a PCS of ESS is installed sufficient size, the effect of PS part is neglected and NMAE is reflected from the residual part of the ESS operation.

The summation of the residual part in (10) does a concave function. This is because the amount of residual is reduced as the operation progresses. In addition, the function in (10) is divided by the length of RTH that is a linear function. It means that NMAE in (10) has an optimal point according to RTH [29]. The Lagrangian multipliers $v_{t}^{-}$and $v_{t}^{+}$only have a value when the ESS operation is restricted by the ES of ESS. Therefore, the optimal RTH is determined by the ES size of ESS and the expected error of onward operations in OTH.

\section{CASE STUDY}

The present study is based on wind-power plants installed in the Columbia River Gorge in U.S. Bonneville Power Administration (BPA) who is a federal Power Market 


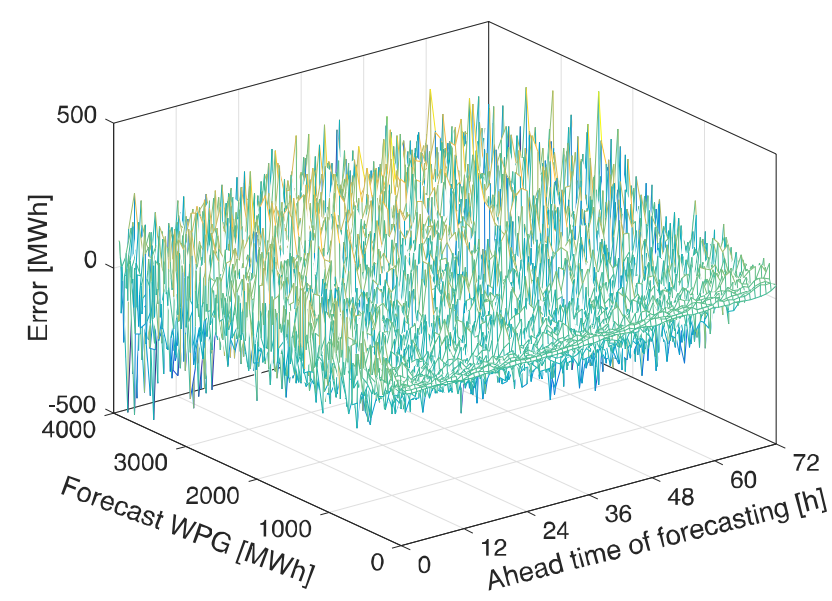

(a) Estimated error

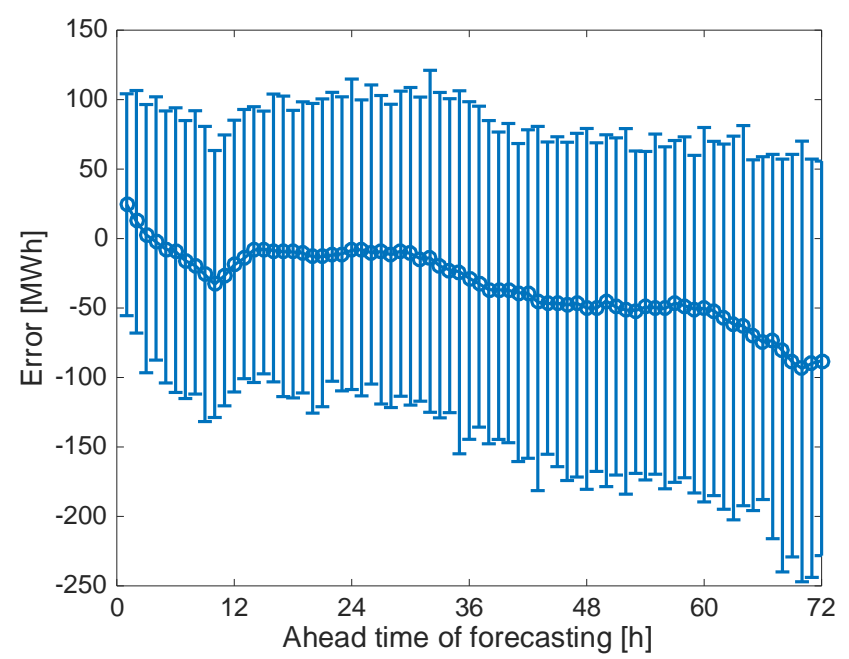

(b) Error distribution according to ahead time of forecasting

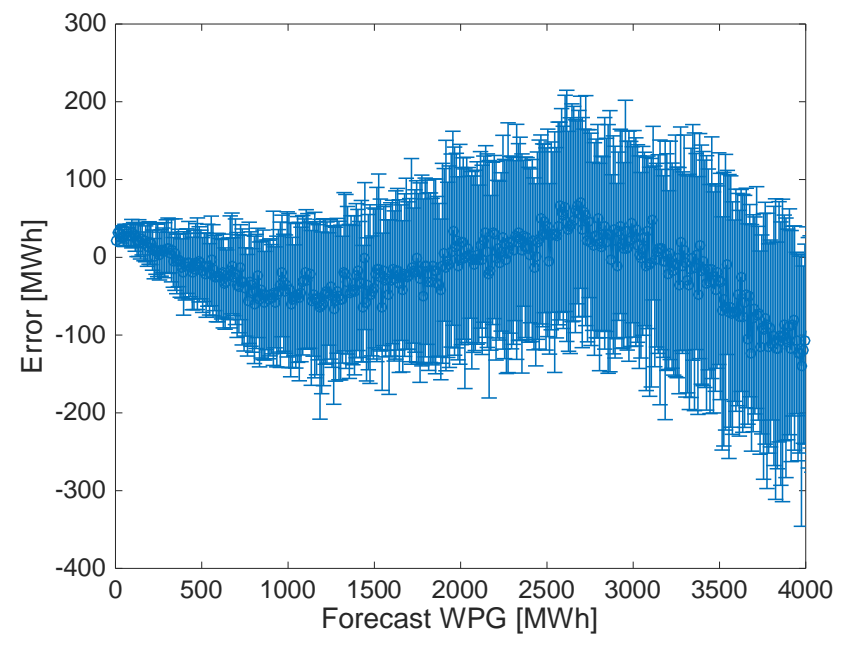

(c) Error distribution according to forecast WPG

Figure 3: Error characteristics according to forecast WPG and ahead time of forecasting. The error bar in Figure 3(b) and 3(c) expresses $70 \%$ confidential bound of the value at each point.
Authority that owns $75 \%$ of the installed transmission in the U.S. Pacific Northwest controls 40 wind-power plants with 4782 MW capacity in 2017 [32]. BPA has announced historical data of WPG and forecasting rolling with every hour. In this study, the data from 2015 to 2017 were used because a plant was added in 2014 .

\subsection{Estimation of Error Part}

To apply the ESS operation in (8), the estimation of error part is required. Using the historical data (the data from 2015 to 2016 in this study), it could be estimated, and the result will apply onward operation with the current forecasting (the data in 2017 in this study). The NMAE which is the objective function is related to the forecast WPG and it is increasing through ahead time of forecasting. Therefore, the error part is estimated according to the forecast WPG and ahead time of forecasting as shown in Figure 3.

Figure 3 shows error characteristics according to forecast WPG and ahead time of forecasting. Figure 3 presets the expected error measured using the historical data. In Figure 3(b) and 3(c), the circled lines mean the average value and the error bar expresses $70 \%$ confidential bound of the value at each point. As shown in Figure 3(a), the expected error has the randomness in forecast WPG and ahead time domain. However, it is observed some trend in Figure 3(b) and 3(c). In Figure 3(b), the error variance illustrated by the error bar is increasing with longer ahead time of forecasting, i.e., about $150 \mathrm{MWh}$ at $1 \mathrm{~h}$ ahead forecasting to $300 \mathrm{MWh}$ at $72 \mathrm{~h}$ ahdaed forecasting. This is because BPA's WPG forecasting is recursively updated using the latest information [33]. In addition, the error has negative values with longer ahead time of forecasting. It means that the forecasting method over estimates WPG. In Figure 3(c), the error variance also grows with increasing forecast WPG, but it is not significant when the forecast WPG is larger than $1500 \mathrm{MWh}$. Therefore, the relative error normalized forecast WPG is reduced increasing forecast WPG. It means that the amount of WPG forecasting is less effect to the error part than the ahead time of forecasting.

The error part is directly measured using the historical data as the value in Figure 3, but, for dynamic ESS operation, it can be modified considering the characteristics,

$$
\hat{e}_{t}=\alpha\left(t, \hat{g}_{t}\right) \times \mathrm{e}_{\mathrm{t}},
$$

where $\alpha\left(t, \hat{g}_{t}\right)$ is the compensation factor considering the error characteristics. A simple example design of the factor is determined related to a reciprocal of the error variance in Figure 3(b) and 3(c).

\subsection{Case Study}

It is considered 450MWh ESS that is about $10 \%$ of total WPG capacity. ESS characteristics are assumed as 90\% round-trip efficiency and 90\% deep-of-death such as Lithium- 


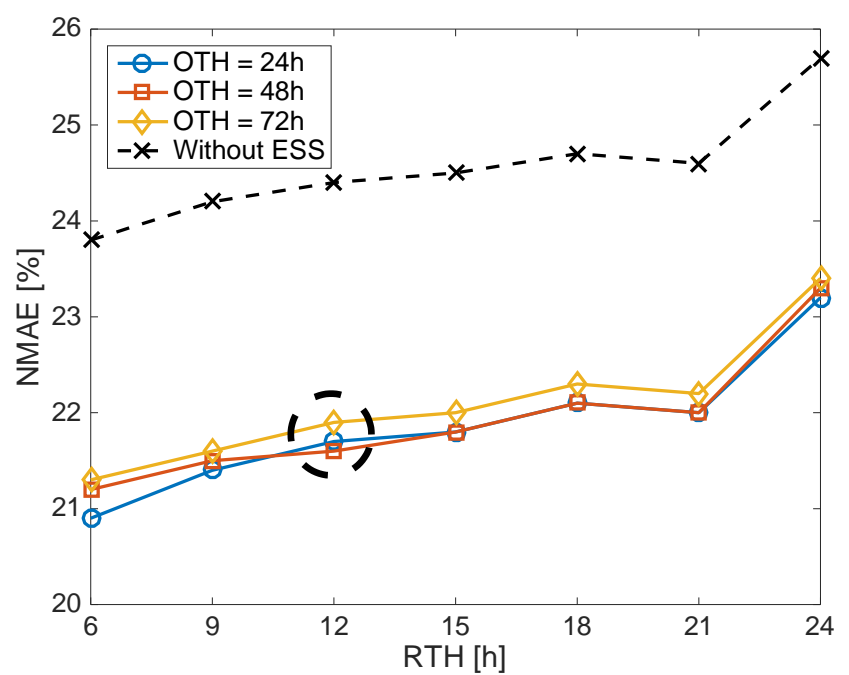

Figure 4: NMAE according to RTH when OTH is $24 \mathrm{~h}, 48 \mathrm{~h}$, and $72 \mathrm{~h}$. The black dashed line expresses the NAME without ESS.

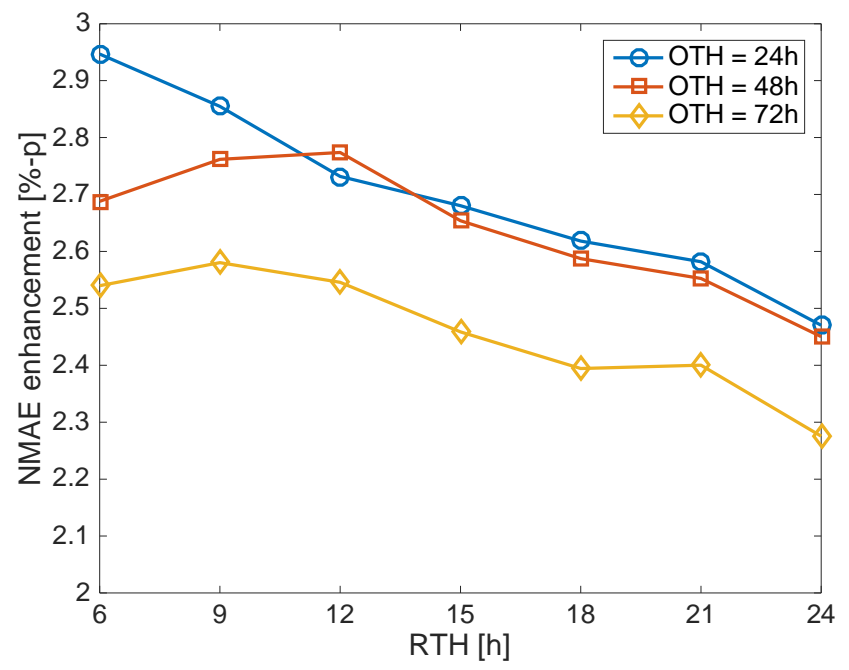

Figure 5: NMAE enhancement compared to that without ESS. It is calculated as percent-point because NMAE is a relative value.

Ion battery that is the state-of-art ESS applying in-front-of-the-meter side [34].

Figure 4 shows NMAE with changing RTH. The lines with circle, square, and diamond presents the value when OTH is $24 \mathrm{~h}, 48 \mathrm{~h}$, and $72 \mathrm{~h}$, respectively. The black dashed line is the NAME without ESS shown for comparison. In all cases, NMAE has the lowest value with the shortest RTH as $6 \mathrm{~h}$. This is because, in more frequent rolling case, the newest forecast WPG is used that has less NAME. For the similar reason, the NAME increases with the longer OTH. However, at the case of short-term OTH, the risk of future cannot be properly reflected as shown in the black dashed circle. In this case, the NMAE with 48h OTH has less value than that with 24h OTH. NMAE is calculated not only the current ESS operation but also the onward ESS operation. The case with 48h OTH more considers the onward ESS operation as the residual part than that with $24 \mathrm{~h}$ OTH. It means that OTH and RTH should be relatively determined considering the characteristic of the WPG forecasting.
Figure 5 shows NMAE enhancement compared to that without ESS that is the black dashed line in Figure 4. NAME is a relative value, so the enhancement is calculated as percent-point [35]. As discussed above, the maximum NMAE enhancement is relatively determined to OTH and RTH. When the short OTH such as $24 \mathrm{~h}$, it is maximized with the short RTH of $6 \mathrm{~h}$. This is because the residual part for the onward ESS operation is small in this case, so the frequent rolling for using the recent information enhances the NMAE. Increasing OTH to $48 \mathrm{~h}$, the NMAE enhancement is maximized at longer RTH of $12 \mathrm{~h}$. It is the point balanced between the current ESS operation and the onward ESS operation. However, in the $72 \mathrm{~h}$ OTH case, the maximum enhancement point is reduced to $9 \mathrm{~h} \mathrm{RTH}$. Because of the longer OTH, the residual part restricts the current operation, thus the rescheduling by rolling is required. It shows that the OTH and RTH is decided related to the operability for the current ESS operation and the onward ESS operation.

\section{CONCLUSION}

This paper proposed the rolling based ESS operation strategy. The goal of this study is to solve the ESS operation problem considering OTH and RTH for managing the wind power forecasting uncertainty. The proposed strategy suggested the solution using Lagrangian multipliers according to the characteristics of the uncertainty and ESS. Numerical results with the actual wind power data show that the ESS applying the proposed strategy effectively manages the wind power uncertainty with about $10 \%$ performance enhancement. Moreover, the relationship between OTH and RTH for the effective ESS operation is presented.

\section{REFERENCES}

1. Statistical Review of World Energy 2018, BP, London, England, 2018.

2. World Energy Outlook 2017, International Energy Agency (IEA), Paris, France, 2017.

3. Global Wind Report 2017, Global Wind Energy Council (GWEC), Brussels, Belgium, 2018.

4. J. Mendes, J. Sumaili, R. Bessa, H. Keko, V. Miranda, A. Botterud, and Z. Zhou. Very short-term wind power forecasting: state- of-the-art, Tech. Rep. ANL/DIS-14/6, Argonne National Lab. (ANL) (Oct. 2014). https://doi.org/10.2172/1158939

5. C. Feng, M. Cui, B.-M. Hodge, and J. Zhang, A data-driven multi- model methodology with deep feature selection for short-term wind forecasting, Applied Energy, Vol. 190, pp. 1245-1257, Mar. 2017.

6. J. R. Andrade and R. J. Bessa, Improving renewable energy forecasting with a grid of numerical weather predictions, IEEE Transactions on Sustainable Energy Vol. 8, No. 4, pp. 1571-1580, Oct. 2017. https://doi.org/10.1109/TSTE.2017.2694340

7. M. Khodayar, and J. Wang, Spatio-temporal graph deep neural network for short-term wind speed 
forecasting, IEEE Transactions on Sustainable Energy, Vol. 10, No. 2, pp. 670-681, Apr. 2019.

8. H. Zhao, Q. Wu, S. Hu, H. Xu, and C. N. Rasmussen, Review of energy storage system for wind power integration support, Applied Energy, Vol. 137, pp. 545-553, Jan. 2015.

https://doi.org/10.1016/j.apenergy.2014.04.103

9. O. Schmidt, A. Hawkes, A. Gambhir, and I. Staffell, The future cost of electrical energy storage based on experience rates, Nature Energy, Vol. 2, No. 8, pp. 1-8, Jul. 2017.

https://doi.org/10.1038/nenergy.2017.110

10. A. Michiorri, J. Lugaro, N. Siebert, R. Girard, and G. Kariniotakis, Storage sizing for grid connected hybrid wind and storage power plants taking into account forecast errors autocorrelation, Renewable Energy, Vol. 117, pp. 380-392, Mar. 2018.

11. A. Abbassi, M. A. Dami, and M. Jemli, A statistical approach for hybrid energy storage system sizing based on capacity distributions in an autonomous $\mathbf{P V} /$ wind power generation system, Renewable energy, Vol. 103, pp. 81-93, Apr. 2017.

https://doi.org/10.1016/j.renene.2016.11.024

12. U. Akram, M. Khalid, and S. Shafiq, Optimal sizing of a wind/solar/battery hybrid grid-connected microgrid system, IET Renewable Power Generation, Vol. 12, No. 1, pp. 72-80, Jan. 2018.

13. N. Ghaffarzadeh, M. Zolfaghari, F. J. Ardakani, and A. J. Ardakani, Optimal sizing of energy storage system in a micro grid using the mixed integer linear programming, International Journal of Renewable Energy Research, Vol. 7, No. 4, pp. 2004-2016, Apr. 2017.

14. E. Oh, and S.-Y. Son, Energy-storage system sizing and operation strategies based on discrete Fourier transform for reliable wind- power generation, Renewable Energy, Vol. 116, pp. 786-794, Feb. 2018. https://doi.org/10.1016/j.renene.2017.10.028

15. Z. Shu, and P. Jirutitijaroen, Optimal operation strategy of energy storage system for grid-connected wind power plants, IEEE Transactions on Sustainable Energy, Vol. 5, No. 1, pp. 190-199, Jan. 2014.

16. Z. Shu, K. T. K. Hock, and G. H. Beng, Operation of energy storage system for renewable utilization enhancement, in: IEEE Power and Energy Society General Meeting (PESGM), Boston, MA, USA, 2016.

17. F. Luo, K. Meng, Z. Y. Dong, Y. Zheng, Y. Chen, and K. $\mathrm{P}$. Wong, Coordinated operational planning for wind farm with battery energy storage system, IEEE Transactions on Sustainable Energy, Vol. 6, No. 1, pp. 253-262, Jan. 2015.

https://doi.org/10.1109/TSTE.2014.2367550

18. Q. Jiang, Y. Gong, and H. Wang, A battery energy storage system dual-layer control strategy for mitigating wind farm fluctuations, IEEE Transactions on power systems, Vol. 28, No. 3, pp. 3263-3273, Mar. 2013.

19. Y. Yuan, X. Zhang, P. Ju, Q. Li, K. Qian, and Z. Fu, Determination of economic dispatch of wind farm-battery energy storage system using genetic algorithm, International Transactions on Electrical Energy Systems, Vol. 24, No. 2, pp. 264-280, Feb. 2014.

20. V. Khare, S. Nema, and P. Baredar, Optimisation of the hybrid renewable energy system by HOMER, PSO and CPSO for the study area, International Journal of Sustainable Energy, Vol. 36, No. 4. pp. 326-343, Apr. 2017.

21. J. J. Roberts, A. M. Cassula, J. L. Silveira, E. da Costa Bortoni, and A. Z. Mendiburu, Robust multi-objective optimization of a renewable based hybrid power system, Applied Energy, Vol. 223, pp. 52-68, Aug. 2018.

22. M. C. Bozchalui, C. Jin, and R. Sharma, Rolling stochastic optimization based operation of distribution systems with PVs and energy storages, in: IEEE PES Innovative Smart Grid Technologies Conference (ISGT), Washington, DC, USA, 2014.

23. H. Ding, Z. Hu, and Y. Song, Rolling optimization of wind farm and energy storage system in electricity markets, IEEE Transactions on Power Systems, Vol. 30, No. 5, pp. 2676-2684, May 2015.

24. R. Palma-Behnke, C. Benavides, F. Lanas, B. Severino, L. Reyes, J. Llanos, and D. Saez, A microgrid energy management system based on the rolling horizon strategy, IEEE Transactions on Smart Grid, Vol. 4, No. 2, pp. 996-1006, Feb. 2013.

25. S. Cordova, H. Rudnick, A. Lorca, and V. J. Martinez, An efficient forecasting-optimization scheme for the intra-day unit commitment process under significant wind and solar power, IEEE Transactions on Sustainable Energy, Vol. 9, No. 4, pp. 1899-1909, Mar. 2018.

https://doi.org/10.1109/TSTE.2018.2818979

26. B. S. Hodge, C. Brancucci Martinez-Anido, Q. Wang, E. K. Chartan, A. R. Florita, and J. Kiviluoma, The combined value of wind and solar power forecasting improvements and electricity storage, Applied Energy, Vol. 214, pp. 1-15, Mar. 2018.

27. Elmer R. Magsino, Energy Monitoring System Incorporating Energy Profiling and Predictive Household Movement for Energy Anomaly Detection, International Journal of Emerging Trends in Engineering Research, Vol. 7, No. 8, pp. 151-156, Aug. 2019. https://doi.org/10.30534/ijeter/2019/08782019

28. A. V. Oppenheim, A. S. Willsky, and S. H. Nawab, Signal and Systems, Pearson Education, London, England, 1996.

29. S. Boyd, and L. Vandenberghe, Convex Optimization, Cambridge University Press, Cambridge, CB2 2RU, UK, 2004.

30. N. Sahebjamnia and A. M. Fathollahi-Fard, A Lagrangian Relaxation-based Algorithm to solve a Collaborative Water Supply Chain Network Design Problem, International Journal of Emerging Trends in Engineering Research, Vol. 6, No. 7, pp. 40-45, Jul. 2018. https://doi.org/10.30534/ijeter/2018/01672018

31. A. A. Akhil, G. Huff, A. B. Currier, B. C. Kaun, D. M. Rastler, S. B. Chen, A. L. Cotter, D. T. Bradshaw, and W. D. Gauntlett, DOE/EPRI 2013 electricity storage handbook in collaboration with NRECA, Tech. Rep. 
Eunsung Oh, International Journal of Emerging Trends in Engineering Research, 7(11), November 2019, 708 - 714

SAND2013-5131, Sandia National Laboratories, Albuquerque, NM, USA (Jul. 2013).

32. Wind generation and total load in the BPA balancing authority, Bonneville Power Administration, U.S. Dept. of Energy.

33. S. Nitsche, C. A. Silva-Monroy, A. Staid, J.-P. Watson, S. Winner, and D. L. Woodruff, Improving wind power prediction intervals using vendor-supplied probabilistic forecast information, in: IEEE Power and Energy Society General Meeting, 2017.

https://doi.org/10.1109/PESGM.2017.8274537

34. Lazard's levelized cost of storage analysis-version 4.0, Tech. rep., LAZARD, New York City, NY, USA, Nov. 2018.

35. R. Brechner, and G. Bergeman, Contemporary Mathematics for Business \& Consumers, Brief Edition, Cengage Learning, Boston, MA, USA, 2015. 